ON THE

STRUCTURE

OF THE

\title{
HUMAN PLACENTA,
}

AND

\section{ITS CONNEXION WITH THE UTERUS.}

Bx WILLIAM BLOXAM, Esq.,

MEMBER OF THE ROYAL COLLEGE OP SURGEONS OF LONDON;

SURGEON TO QUEEN ADELAIDE'S LYING-IN-HOSPITAL;

AND LECTURER ON MIDWIFERY AT THE SCHOOL OF ANATOMY AND MEDICINE ADJOINING ST. GEORGE'S HOSPITAL.

READ MARCH 24TH, 1840.

IN offering the following observations to the notice of this Society, I will take the liberty of stating that my attention was drawn to this important and interesting subject, principally in consequence of the contradictory opinions which have been promulgated by individuals of the highest character, in reference to the intimate structure of the placenta.

Finding that the perusal of the greater part of the writings on the subject tended to leave the mind in the same unsettled state in which the study was commenced, I determined to spend some time in investigating the various points connected with it; 
resolving, as far as was in my power, to preserve myself in perfect freedom from prejudice or preconceived ideas, and to be contented with becoming a faithful reporter of facts.

These examinations have extended over a considerable period, during which time every separate step has been again and again repeated.

Having carefully examined the structure of the placenta, I ventured to compare the results, at which I had arrived, with the investigations of others; and as may be naturally anticipated, many points which I considered new, were either wholly, or in part, known. There are, however, one or two other points, which, as far as I am aware, are not yet recorded, if known.

Convinced, as I am, that our knowledge of the structure and functions of this organ is exceedingly imperfect, and that there is yet ample field for future inquiry, I may be considered guilty of presumption, in offering the following remarks to the notice of the Society, particularly as I lay no claim to originality. Yet, with those who are acquainted with the difficulties of minute anatomy, I shall stand acquitted, should it be found that $I$ have brought forward any evidence corroborative of the investigations of others, or have thrown out a single hint which may smooth the path of my fellow-labourers on the subject.

The only merit to which I venture to lay claim, is some degree of patience, and also fidelity, in recording what I have observed.

VOL. XXIII. 
An opportunity was afforded me, some time past, of examining the uterus of a female, who died about thirty hours after a most severe labour.

When removed from the body, its volume equalled that of the impregnated uterus between the fourth and fifth month; and it was of an intensely red colour, almost amounting to the tint known by the name of Modena red, and approaching a state of decomposition. On dividing it perpendicularly from the fundus on its fore part, the inner surface was found to be completely covered by a coat of grumous blood. It was then carefully immersed in a vessel of water, and agitated from time to time; a fresh supply of water being poured on it till it ceased to become turbid.

The inner surface presented the following appearances, which are to be observed in Plate III., engraved from a drawing made by Mr. Perry, with his usual fidelity and skill.

The muscular fibres of the uterus, at those parts to which the placenta had not been attached, were perfectly visible in rounded columns, not unlike those of the carneæ columnæ of the heart; and no orifice of any description was to be seen on the most accurate examination.

At the upper and back part of the fundus, was a space, irregularly circular, of from six to seven inches in diameter, of a darker colour than the rest, to which the placenta had evidently been attached. This space was completely covered by a delicate flocculent membrane. 
On carefully turning this membrane about, with a fine needle, numerous openings were observed in it, having a well-defined margin, and forming canals leading to the substance of the uterus. Some of these canals are sufficiently large to admit the little finger.

At the bottom of these canals may be observed openings on the surface of the uterus, having a welldefined edge, either of an elliptic or semilunar form, penetrating obliquely downwards into the substance of the organ: some of these, also, are from four to five, or even six lines in their long diameter.

I have now described two sets of openings. But there is also a third, which may be observed on looking into thosé last mentioned, when they will be seen of similar form, differing, however, in size, and, generally, much smaller. The direction of the channels into which they lead is, also, from above downwards; and it is particularly to be observed, that no two openings are upon the same meridian.

These openings lead into the large uterine sinuses, and the course of one of them is indicated by a piece of bougie, as seen in Plate III.

This sinus is lined by a smooth membrane, resembling that of the larger veins of the trunk, and is triangular in shape; the apex being turned towards the inner, and the base towards the outer wall, of the uterus. At its posterior angles, on each side, it receives the trunks of the smaller veins, in pairs, at distances of about nine lines apart; and at 
its inferior portion, becomes continuous with the hypogastric veins.

It will now be necessary to refer to the placenta. But its structure at the completion of utero-gestation being the principal object of this paper, it is not my intention to enter on the question of its early development.

If a placenta which has been excluded by the natural efforts of the uterus be freed from the coagula attached to its uterine surface by gentle agitation in warm water, vessels of very delicate texture, varying in size from an extreme tenuity to the calibre of a line, will be found on its uterine surface.

The length of these vessels, (which from the irregular form of their free extremity are evidently broken off,) varies from two to six or eight lines; and, as far as I have observed, they are invariably found with a depressed line in their longitudinal axis, as we generally see the arteries after death.

In Plate IV. fig. 1, the subject of which was taken from the body of a phthisical patient, who died at the sixth month of utero-gestation, these vessels may be observed crossing from the uterus to the placenta. They are of small diameter, but this circumstance may probably be, in some measure, the consequence of the early period at which gestation had been arrested.

These vessels, which after making a complete circle, plunge suddenly into the surface of the placenta, are known by the name of the short curling arteries of Hunter. Their ultimate distribution will 
be considered hereafter; this much may, however, be stated at this stage of the inquiry ; that they are most probably the sources by which the blood enters the placenta, for the nourishment of the child.

The same vessels are beautifully shown in a preparation in the gallery of the museum of the Royal College of Surgeons, No. 3,540.

If we now place the placenta on a level surface, with its uterine side uppermost, a number of openings may be detected.

These openings are well seen in the wax cast of a placenta in my possession. In this cast, the edges of the openings have been slightly defined with black varnish, so as to distinguish them from the adjacent surface; and where the orifice is large, and has penetrated some considerable depth into the placenta, the bottom of the aperture has also been made black, so as to render it visible. Some of the openings approach the elliptic, some the circular form, whilst others, and the great majority of them, are semilunar.

These last-named openings penetrate obliquely the substance of the organ; and inflation of one of them will distend the whole of the lobe to which it belongs, and frequently a considerable portion of the lobes adjacent.

It is remarkable that the concavity of every one of these semilunar valves, for so they may be called, is turned, in some degree, towards the insertion of the cord.

Passing round the circumference of the placenta, is to be seen a sort of canal, Plate IV. fig. 2, lined 
by a membrane exactly resembling in appearance the inner coat of a vein.

In this canal are many apertures, with semilunar openings directed in the same manner as the semilunar openings of the placental mass itself; and it receives numerous trunks of veins coming, apparently, from the membranes of the ovum.

Several of these openings have a bristle inserted into their mouths; and one of them is distended by a bristle which has been doubled.

The smooth lining membrane may be seen immediately beneath the bougie which has been introduced into the canal.

It may now be stated that in place of the placenta being of cellular structure, such is not the fact. If a vertical section be examined under water, it will be found to be composed of vascular structures arranged in the form of convolutions, which have given this erroneous impression; the cells being merely the interstitial spaces of the vascular arrangement, and presenting nothing in any degree resembling cavities with definite parietes.

Into this interstitial portion of the placenta, the semilunar apertures on the uterine surface of the organ lead, as may be seen in Plate V. fig. 1, where tallow coloured with carmine has been injected from one of these apertures.

I have repeatedly injected the interstices of an entire placenta from one or other of these orifices.

From the oblique mode in which they penetrate the organ, they appear to possess somewhat of the 
action of a valve, as I am induced to believe they almost entirely resist the passage of fluids from the system of the mother towards that of the child: and in the gravid uterus already mentioned, size thrown into the spermatic arteries freely penetrated, (as may be seen in Plate IV. fig. 1,) the vascular system of the placenta; rendering it of a uniform redness, except under the highest magnifying power : whilst size, coloured blue with finely levigated smalt, was injected into the hypogastric veins, none has appeared to have reached the placenta; notwithstanding it can easily be traced in the sinuses of the uterus.

The probable inference, therefore, is, that the current of blood does not enter the placenta through those orifices.

It will be now necessary to refer to the distribution of the vessels called the curling arteries of Hunter, which appear to be the way by which the blood, or its nutritive principle, is conveyed to the placenta.

As has been already remarked, they are exceedingly numerous; of small calibre compared with the important function they perform, and from this fact I shall venture hereafter to deduce a conclusion of considerable consequence.

These vessels, immediately on their entrance into the placenta, appear to divide abruptly into an extreme number of smaller branches, so small, as to be perfectly undistinguishable by the naked eve; and even under a simple lens of high focus a sec- 
tion of the placenta appears of an uniform colour; but under the microscope, they present perfect evidence of linear arrangement. These vessels appear to ramify very minutely in the convolutions of the placenta.

A vertical section of the organ presents them as a number of extremely minute points, and a magnified view of a small portion of placenta may be seen in Plate V. fig. 2.

With reference to the distribution of the umbilical vein and artery, the following circumstances may be observed.

It appears to me, from injection, and from the examination of the preparations in the various museums of this metropolis, that no injection has hitherto reached the vessels of the cord from the uterine arteries; it may be therefore inferred, that there is no actual communication with them by continuity of structure; but on observing the distribution of the umbilical vein at its extremities, it is found that its divisions are excessively minute, and in contact with the branches of the uterine arteries, ramifying also on the spongy tissue of the placenta.

As an injection thrown with extreme care into the umbilical vein will return oecasionally by the arteries, the argument is apparently conclusive, that there is a union of these vessels, by means of a capillary system as well as an inosculation between the minute branches of the umbilical vein itself.

The umbilical artery has also two modes of termination; one, with the capillary system above men- 
tioned, and a second, which I am not aware has been previously observed.

If the placenta be carefully examined under spirit, and the laminæ of the spongy tissue gently drawn asunder, the extreme branches of the umbilical arteries may be seen floating in the liquid, between the convolutions of the organ.

If one of these terminal branches be carefully detached, and received on a piece of glass under the spirit, and viewed by the microscope, its termination will be found as in Plate V. fig. 3. It seems to terminate in several tufts of vessels, bulbous at their free extremity, into which the colouring matter of the injection very imperfectly enters ; but which, by occasional dots of vermillion, are proved to be prolongations of the canal of the vessel.

A singular peculiarity is observable in these vessels; namely, that they almost invariably form a loop resembling a figure of eight.

In those portions of placenta in which I have injected the interstitial structure with tallow from the semilunar valves of the uterine surface, $I$ have been able, on melting the tallow, by pouring boiling alcohol upon a thin vertical section, to elevate these tufts of vessels on the point of a fine needle, in every instance; although they are so minute as not to be distinguishable without the aid of a good lens.

In reviewing these facts, the following conclusions appear to me deducible.

Firstly. That the blood enters the placenta through 
the short curling arteries of Hunter; and that although their size is insignificant compared with the venous system of the uterus, yet their relative number being greater, a full supply of blood is insured to the organ; whilst by the smallness of their calibre, they prevent the maternal circulation from expending its full momentum on the system of the child, under the accidental shocks physical and mental to which the mother is daily liable.

Secondly. That these vessels ramify on the spongy tissue of the placenta, and are there in apposition with the extremities of the umbilical veins.

Thirdly. That it is highly probable, that some of the properties of the maternal blood pass into the circulation of the fœtus by this means, and having fulfilled its functions in the fœtal œconomy, the residue is returned to the placenta by the umbilical artery.

Fourthly. That from the free terminations of the umbilical arteries it transudes into the interstitial structure of the placenta ; which, it may be remembered, is continuous with the semilunar apertures on its uterine surface.

Fifthly. That these apertures are applied to the openings on the internal surface of the uterus, and furnish the channel by which the blood, or its principles, are restored to the system of the mother.

As far as I am aware, there is no evidence of direct communication between the foetus and its parent, furnished by the researches of comparative anatomists. Mercury, however, has been found 
in the vessels of the fœtus after having been injected from the uterus. Here, perhaps, the weight of the substance employed may have conduced to the result.

In the preparation from which the drawing of Plate IV. fig. 1 , is taken, it will be observed, that the injection of blue-coloured size, which has freely penetrated the venous system of the uterus, has not entered the substance of the placenta, although the openings of the venous channels are many times larger than those of the arterial system, which is minutely injected.

Was this injection arrested by the semilunar valves of the uterine surface of the placenta? It appears to me very probable.

With regard to the precise process which the blood undergoes in its accommodation to the necessities of the fotus, we have no data to guide us ; it may be a secretory process, or merely an exercise of those peculiar properties of the vascular system known as endosmose and exosmose.

I. cannot conclude this paper without a brief observation on the mechanical arrangement of the uterine orifices for the prevention of hæmorrhage.

In regarding the immense size of the apertures of the venous sinuses, as in Plate I., one feels a degree of astonishment that hæmorrhage is not more frequent, and more fatal ; but when it is remembered that the essential property of the uterine fibre, at the full term of gestation, is that of contractility, and that by the exercise of this property the 
orifices of the deeper seated sinuses must be closed, by the more internal layer of muscular fibres, inasmuch as these apertures are not placed on the same meridian, our surprise ceases.

It is probable, that independently of this mechanical arrangement, the flow of blood from the more superficial openings may be arrested by the formation of coagula, a process for which the flocculent structure of the decidua seems highly favourable. 


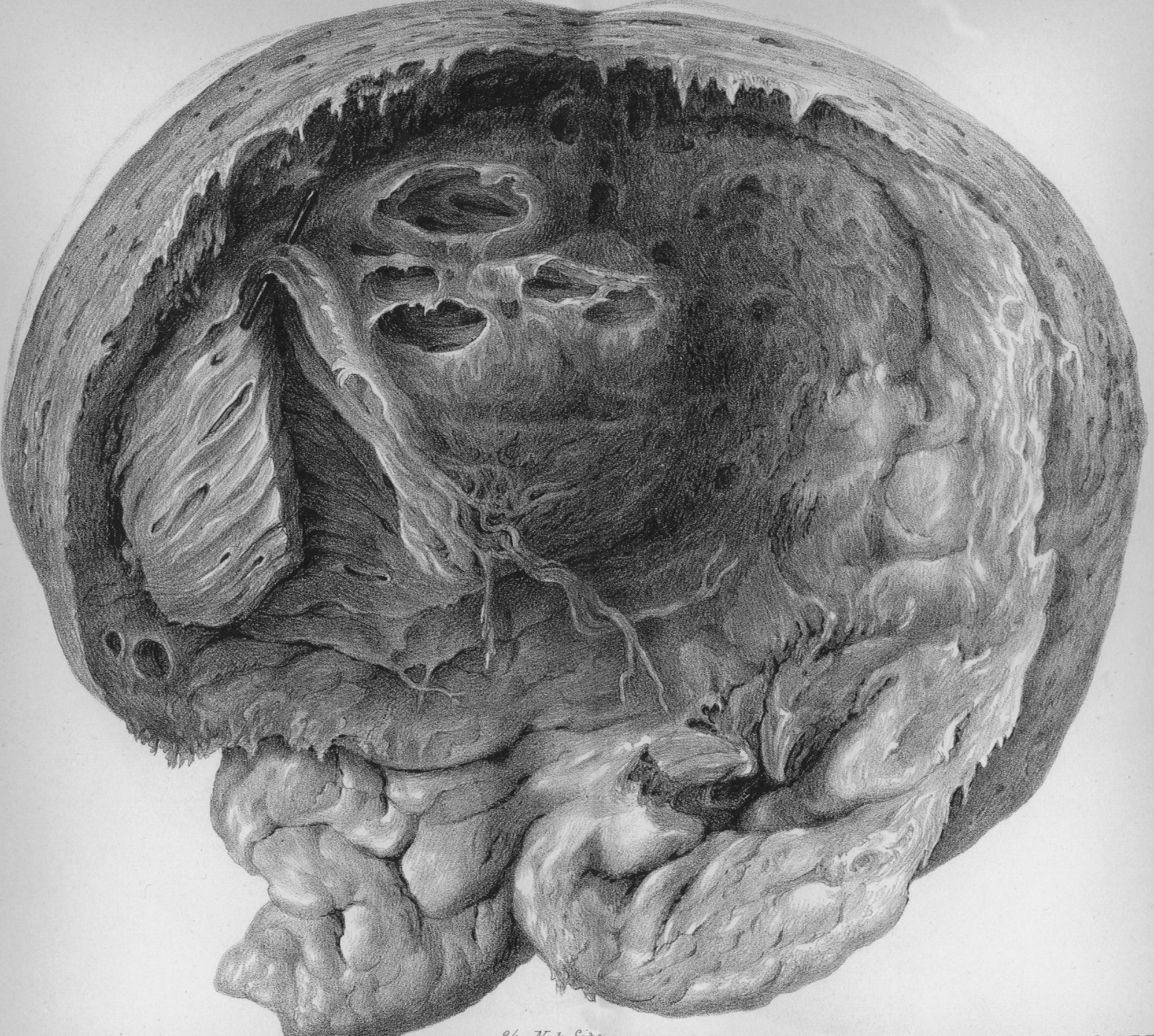



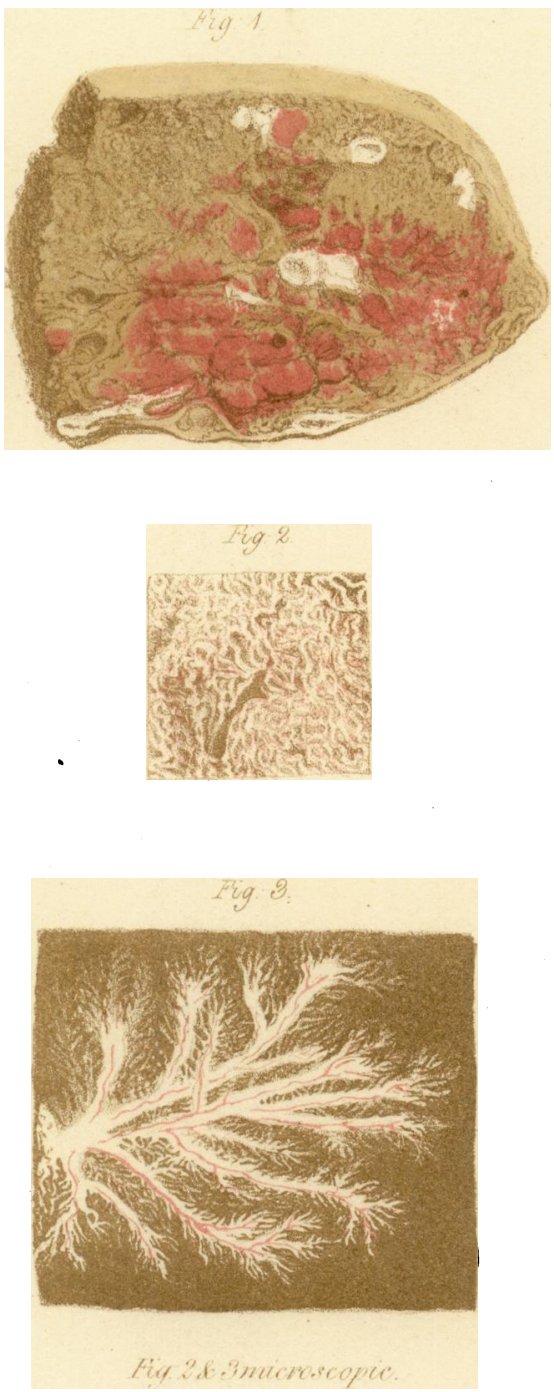

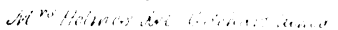



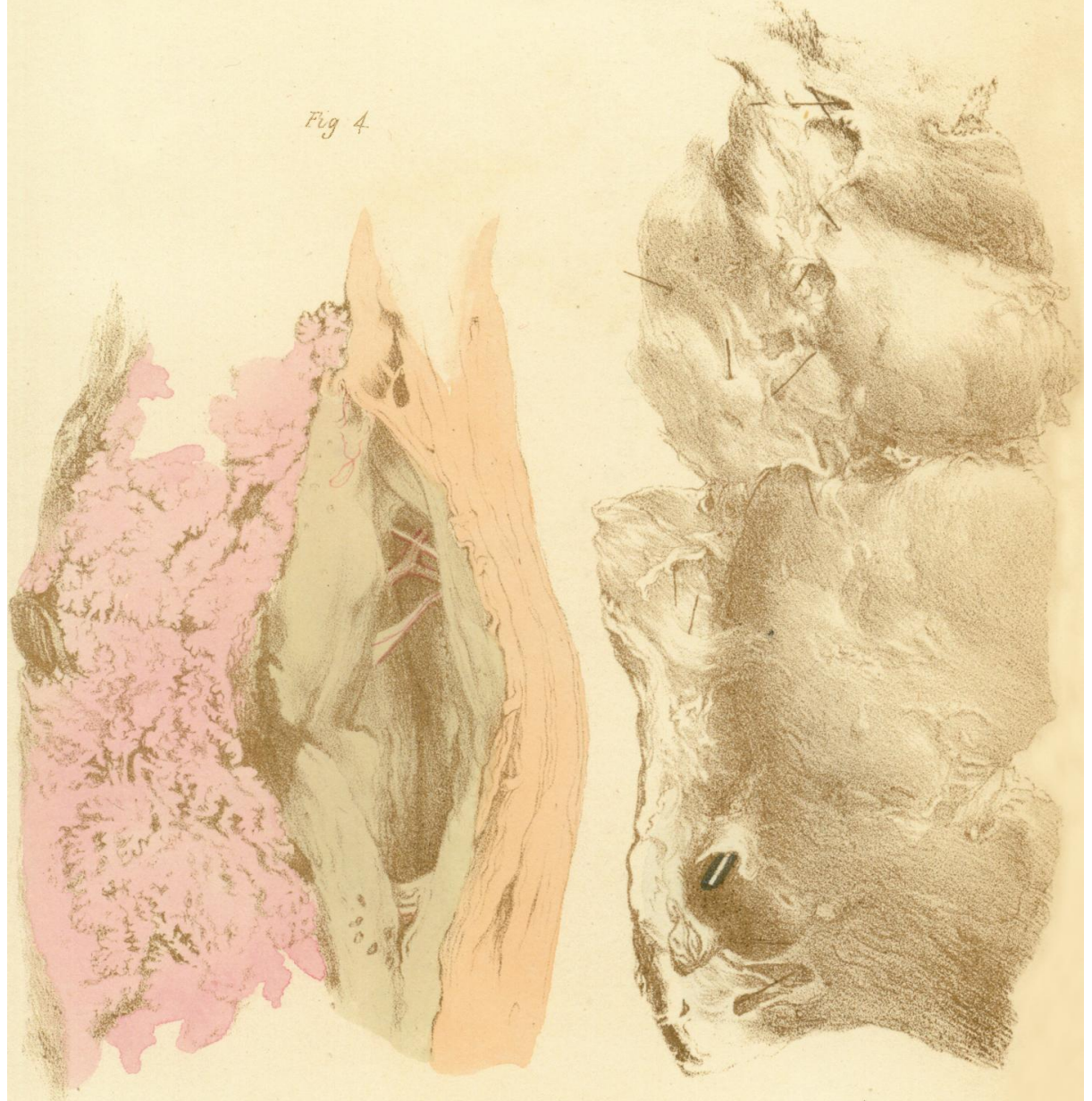